\title{
Connectivity, Graph Minors, and Subgraph Multiplicity
}

\author{
David Eppstein \\ Department of Information and Computer Science \\ University of California, Irvine, CA 92717
}

January 5, 1993

\begin{abstract}
It is well known that any planar graph contains at most $O(n)$ complete subgraphs. We extend this to an exact characterization: $G$ occurs $O(n)$ times as a subgraph of any planar graph, if and only if $G$ is three-connected. We generalize these results to similarly characterize certain other minor-closed families of graphs; in particular, $G$ occurs $O(n)$ times as a subgraph of the $K_{b, c}$-free graphs, $b \geq c$ and $c \leq 4$, iff $G$ is $c$-connected. Our results use a simple Ramsey-theoretic lemma that may be of independent interest.
\end{abstract}

\section{Introduction}

It follows from the sparsity of planar graphs that each such graph contains at most $O(n)$ complete subgraphs $K_{3}$ and $K_{4}$ [6]. All cliques in a planar graph can be listed by an algorithm with $O(n)$ worst-case time complexity [3, $4,6]$. Enumeration of subgraphs has a number of uses, including a recent application in testing inscribability [5].

These results naturally raise the question of determining which other planar graphs occur $O(n)$ times as subgraphs of planar graphs. A necessary condition is that the subgraph $G$ be 3 -connected: otherwise, $G$ can be split into two parts $A$ and $B$ by a separating pair of vertices, and by connecting many copies of $A$ and $B$ at the same two vertices, we find a family of planar graphs in which $G$ occurs $\Omega\left(n^{2}\right)$ times as a subgraph.

Our main result is that this is also a sufficient condition. Every threeconnected planar graph can only occur $O(n)$ times as a subgraph of other planar graphs. 
We extend this to minor-closed families of graphs. As is well known, the planar graphs are exactly those for which neither $K_{3,3}$ nor $K_{5}$ is a minor. We first investigate graphs for which some complete bipartite graph $K_{b, c}$ (with $b \geq c$ and $c \leq 4$ ) is not a minor. We show that the subgraphs occurring $O(n)$ times in this family of graphs are exactly the $c$-connected graphs. We extend this to other minor-closed families of graphs where some complete bipartite graph is excluded. Our main result for planar graphs appears as a corollary of this generalization.

Our proofs use a simple Ramsey-theoretic lemma that may be of independent interest. In English, it states that if a set is formed as the union of a large number of $k$-tuples, then we can find a smaller set of tuples for which each member of the set always appears in the same tuple position. In the language of graph theory, if many copies of a subgraph occur in a larger graph, we can find a smaller number of copies of the subgraph, so that among those copies each vertex of the larger graph appears in only one way as a vertex of the subgraph.

Finally, we discuss algorithmic enumeration of subgraphs. In some cases, we are able to make our results constructive, so that the copies of a given subgraph can be enumerated in $O(n)$ time, matching the previous results for cliques in planar graphs.

\section{A Ramsey-theoretic lemma}

Given a set $S$, denote the ordered $k$-tuples of members of $S$ by $S^{k}$. We do not allow any tuple to contain the same element in more than one position. Each tuple can be interpreted as a function from the integers $1 \ldots k$ onto the set $S$, such that for any tuple $t$, and $1 \leq i<j \leq k, t(i) \neq t(j)$.

Suppose we have a collection $T$ of $k$-tuples, $T \subset S^{k}$. We say $T$ is coherent if each member of $S$ appears in at most a single position of the $k$-tuples. In other words, for any distinct tuples $t$ and $t^{\prime}$, and for all integers $1 \leq i<i^{\prime} \leq k, t(i) \neq t^{\prime}\left(i^{\prime}\right)$.

As we now show, any set of tuples has a large coherent subset.

Lemma 1. Suppose we are given a set $T$ containing at least $k !^{2} m^{k} k$-tuples. Then there is a coherent subset $T^{\prime} \subset T$ containing at least $m$ tuples.

Proof. We use induction on $k$. Any set of 1-tuples is coherent.

First assume some element $x \in S$ is contained in at least $k !^{2} m^{k-1} / k$ of the $k$-tuples. Then there is a collection of $(k-1) !^{2} m^{k-1}$ of those tuples for which $x$ always appears in some particular position, say position $k$. By 
ignoring this shared position, we construct a collection of $(k-1)$-tuples

which by induction must have a coherent subcollection of size $m$. Restoring the shared positions gives our coherent subset $T^{\prime}$.

Otherwise, each element is in fewer than $k !^{2} m^{k-1} / k$ of the tuples. Then we can repeatedly pick a tuple $t$, add it to $T^{\prime}$, and throw away the tuples that have an element in common with $t$. By the time we run out of tuples to pick, we will have added $m$ tuples to $T^{\prime}$.

\section{Complete bipartite minors}

We first show that not too many copies of a highly connected subgraph can share the same low-degree vertex, unless the graph contains a large complete bipartite graph as a minor. The bound on the number of times the subgraph can occur will then follow from the sparseness of minor-free graphs.

Lemma 2. Let $G$ be a c-connected graph with $k$ vertices. Suppose that $k !^{2} b^{k^{2}} d^{c k^{2}}$ copies of $G$ are subgraphs of some larger graph $H$, and that some vertex $x$ (with degree $d$ in $H$ ) is contained in each of these copies of $G$. Then $H$ contains a complete bipartite graph $K_{b, c}$ as a minor.

Proof. By Lemma 1, we can find $b^{k} d^{c k}$ copies of $G$ such that each vertex of $H$ is used in at most one way as a vertex of $G$. We throw away the remaining copies of $G$.

We now have a one-to-many correspondence between the vertices of $G$ and those of $H$. Suppose each vertex of $G$ corresponds to fewer than $b d^{c}$ different vertices of $H$. Each copy of $G$ could then be found by choosing one such vertex of $H$ for each of the $k$ vertices of $G$, so there would be fewer than $\left(b d^{c}\right)^{k}$ possible copies of $G$ in the coherent set, a contradiction. Therefore we can find some vertex $y$ of $G$ that corresponds to at least $\left(\begin{array}{l}d \\ c\end{array}\right)(b-1)+1<b d^{c}$ vertices of $H$.

Since $G$ is $c$-connected, we can find $c$ vertex-disjoint paths in $G$ from $y$ to $x$. Partition the remaining copies of $G$ into equivalence classes according to the edges in $H$ adjacent to $x$ used by these paths. There are $\left(\begin{array}{l}d \\ c\end{array}\right)$ such classes, so some class uses at least $b$ copies of vertex $y$. Unless $b=1, y$ will not be adjacent to $x$ in $G$; if $b=1$ the lemma is trivially solved by the neighbors of any vertex. We select the copies of $G$ in this class, and throw away the remaining copies.

We then form a minor of $H$ by removing all edges not corresponding to portions of these vertex-disjoint paths, and contracting all remaining edges except those adjacent to $x$ or to copies of $y$. Because the paths are 
vertex-disjoint in $G$, and because we have selected a coherent collection of subgraphs, the images of the paths in $H$ are also vertex-disjoint. Thus the contraction process described above finds a minor in which $x$ is connected to $c$ vertices, which are also each connected to $b-1$ copies of $y$. This gives us $K_{b, c}$ as a minor of $H$.

Theorem 1. Let $F_{b, c}$ be the family of graphs in which $K_{b, c}$ does not occur as a minor, and let $G \in F_{b, c}$ be c-connected. Then there is some constant $\kappa=\kappa(G)$ such that, for any n-vertex graph $H \in F_{b, c}$, there can be at most $\kappa n$ copies of $G$ as a subgraph in $H$.

Proof. Since $F_{b, c}$ is a minor-free family of graphs, the graphs in $F_{b, c}$ are sparse (any such graph has $O(n)$ edges). Thus we can find a vertex $x$ in $H$ which has degree at most some constant $d=O(1)$. By Lemma 2, there are at most $O(1)$ copies of $G$ as a subgraph of $H$ containing vertex $x$. Let $\kappa$ denote this number of copies. Then by induction there are at most $\kappa(n-1)$ copies of $G$ in the graph $H^{\prime}$ formed by removing $x$ from $H$. Thus there are at most $\kappa n$ copies in all.

\section{Other forbidden minors}

We have shown that $c$-connected graphs cannot occur more than linearly within the $K_{b, c}$-free graphs. We now show that for small $c$ this is an exact characterization: graphs with smaller connectivity will always be able to occur more than a linear number of times. We also generalize this characterization to some other minor-closed families of graphs.

Theorem 2. Let $S$ be a set of c-connected graphs containing some complete bipartite graph $K_{b, c}$, with $b \geq c$ and $c \leq 3$. Denote by $F_{S}$ the family of graphs not containing any member of $S$ as a minor. Then a graph $G \in F_{S}$ occurs $O(n)$ times as a subgraph of members of $F_{S}$ if and only if $G$ is c-connected.

Proof. If $G$ is $c$-connected, the result follows from Theorem 1 and the fact that $F_{S}$ is a subset of $F_{b, c}$.

If $G$ is in $F_{S}$ but not $c$-connected, it can be separated by some (c-1)-tuple of vertices. Let $A$ and $B$ be two components of the separated graph. Then we can form a family of graphs in which $G$ occurs $\Omega\left(n^{2}\right)$ times, by connecting many copies of $A$ and $B$, together with a single copy of $G-A-B$, to the same $(c-1)$-tuple. We now show that no such graph $G^{\prime}$ contains a minor in $S$. 
We assume for a contradiction that such a minor, $M$, exists. $M$ is formed by contracting certain connected sets of vertices in $G^{\prime}$, and then possibly removing some edges. First note that no two such sets can be entirely contained within components of $G^{\prime}$ on different sides of the separating $(c-1)$ tuple, since the two corresponding vertices in $K_{b, c}$ would then be separated by a cut of at most $(c-1)$ vertices. So all vertices in the $K_{b, c}$ minor come from sets containing at least one vertex in some particular component $C$ of $G^{\prime}$. At most $(c-1) \leq 2$ vertices in $M$ can come from sets involving points outside that component, because each such set must include a vertex in the separating $(c-1)$-tuple. So the portion of $G^{\prime}$ outside $C$ can only contribute a single edge in $M$, connecting those two vertices. But that same edge can be found in $G$ by contracting one of components $A$ and $B$ (whichever is not equal to $C$ ), so $G$ also contains $M$ as a minor, contradicting the assumption that $G$ is in $F_{S}$.

Corollary 1. An outerplanar graph $G$ occurs $O(n)$ times as a subgraph of all outerplanar graphs if and only if $G$ is biconnected.

Proof. Apply Theorem 2, with $S=\left\{K_{2,3}, K_{4}\right\}$.

Corollary 2. A planar graph $G$ occurs $O(n)$ times as a subgraph of all planar graphs if and only if $G$ is 3-connected.

Proof. Apply Theorem 2, with $S=\left\{K_{3,3}, K_{5}\right\}$.

We can extend the theorem to a more restricted class of $K_{b, 4}$-free graph families:

Theorem 3. Let $S$ be a set of triangle-free 4-connected graphs containing some complete bipartite graph $K_{b, 4}$, with $b \geq 4$. Denote by $F_{S}$ the family of graphs not containing any member of $S$ as a minor. Then a graph $G \in F_{S}$ occurs $O(n)$ times as a subgraph of members of $F_{S}$ if and only if $G$ is 4connected.

Proof. Sufficiency follows from Theorem 1. For necessity, we follow the previous proof: we assume $G$ has a separating 3 -tuple, and repeat certain components around this tuple to construct a family of graphs $G^{\prime}$ in which $G$ occurs $\Omega\left(n^{2}\right)$ times. Let $M$ be a triangle-free minor of some such graph $G^{\prime}$. As before, all vertices of $M$ come from sets involving a single component $C$ of $G^{\prime}$, and at most three can cross the separating 3-tuple. Then since $M$ is triangle-free, one of the three vertices is adjacent to all edges among the three. By collapsing all of $G$ outside of $C$ into that one vertex, we find $M$ as a minor in $G$ as well as in $G^{\prime}$, so $M$ cannot be in $S$. 
Unfortunately the same proof technique runs into trouble for $K_{b, 5}$-free graphs: there are graphs containing no $K_{5,5}$-minor, yet having a separating 4 -tuple for which any repetition of components as above would form a $K_{5,5^{-}}$ minor.

\section{Algorithms}

We now discuss algorithms for enumerating the occurrences of a given subgraph. Clearly, for any fixed subgraph $G$, there is a polynomial time algorithm for enumerating its occurrences: if there are $k$ vertices in $G$, simply test all $\left(\begin{array}{l}n \\ k\end{array}\right)=O\left(n^{k}\right)$ possible choises for those vertices. We are interested in situations in which the occurrences can be listed more efficiently, in linear time (matching our bounds on the occurrences of $G$ ).

Such algorithms were already known for enumerating copies of the complete graphs $K_{3}$ and $K_{4}$ as subgraphs of planar graphs. More generally, in any family of graphs with bounded arboricity, all clique subgraphs can be listed in linear time $[3,4]$.

The family of $K_{1, b}$-free graphs is also relatively easy. Since these graphs have maximum vertex degree $b-1$, there are fewer than $b^{k}=O(1)$ vertices within distance $k$ of any given vertex. The occurrences of any connected subgraph $G$ can therefore be enumerated in time $O(n)$.

The first interesting cases are the families of $K_{2, b}$-free graphs. Theorem 1 shows that in any such family, the subgraphs appearing $O(n)$ times are the biconnected graphs. The biconnected $K_{2,2}$-free graphs are simply the triangles, which can be enumerated using the clique algorithms cited above. We next consider $K_{2,3}$-free graphs. The biconnected components of these graphs are either outerplanar graphs, or copies of $K_{4}$. Thus we can enumerate any biconnected subgraph of such a graph, if only we can do so for outerplanar graphs.

Theorem 4. Let $G$ be biconnected. Then there is a linear-time algorithm which enumerates the occurrences of $G$ in any outerplanar graph.

Proof. Any biconnected outerplanar graph is Hamiltonian, and adding extra edges to a graph only makes it easier to enumerate its occurrences, so we can assume without loss of generality that $G$ is a simple cycle $C_{k}$. Let $H$ be an outerplanar graph with $n$ vertices, for which we are to enumerate the occurrences of $G$ as a subgraph.

Any occurrence of $G$ consists of the vertices in a union of facial cycles of $H$. To find an occurrence of $G$, we proceed as follows. We start by choosing 
a single facial cycle in $H$. Then as long as the current subgraph is a cycle shorter than $G$, we choose an edge of the subgraph and replace it by the adjacent facial cycle. There are at most $k$ edges to be replaced at each step, and each step increases the cycle length by at least one, so there are $O\left(k^{k}\right)$ ways to augment the initially chosen cycle into an occurrence of $G$. Thus the total time to enumerate the occurrences of $G$ is $O\left(k^{k} n\right)=O(n)$.

Corollary 3. Let $G$ be biconnected. Then there is a linear-time algorithm which enumerates the occurrences of $G$ in any $K_{2,3}$-free graph.

Corollary 4. Let $G$ be a wheel $W_{k}$. Then there is a linear-time algorithm which enumerates the occurrences of $G$ in any $K_{1,2,3}$-free graph, and in particular in any $K_{3,3}$-free or planar graph.

Proof. $W_{k}$ consists of a single "hub" vertex connected to each vertex of a cycle $C_{k}$. Let $H$ be a $K_{1,2,3}$-free graph. We independently enumerate, for each vertex $x$ of $H$, the occurrences of $W_{k}$ for which $x$ corresponds to the hub. This can be done by finding all occurrences of $C_{k}$ in the neighbors of $x$. But the induced graph of these neighbors must be $K_{2,3}$-free, as any $K_{2,3}$ minor together with the hub vertex would form a $K_{1,2,3}$ in the original graph. So by Corollary 3 we can find all such occurrences in time linear in the number of neighbors. The time to find all occurrences of $W_{k}$ is proportional to the sum of the degrees of each vertex of $H$, which is $O(n)$.

This result generalizes the enumeration of cliques in planar graphs, since the only such cliques that can occur are $K_{3}$ and $K_{4}$, which can alternately be interpreted as wheels $W_{2}$ and $W_{3}$.

We would like to be able to extend our results further, at least to the enumeration of all 3-connected planar subgraphs. We have been unable to achieve such a result. However Corollary 4 is promising, as wheels figure prominently in Tutte's characterization of triconnected graphs [7].

\section{Conclusions}

We have described a number of circumstances in which some subgraph occurs $O(n)$ times in the graphs of a minor-closed family. We have also described algorithms that, in a more limited class of situations, can efficiently enumerate the occurrences of a given subgraph.

A number of directions for possible generalization suggest themselves. First, we would like to extend our algorithms so that we can enumerate 
the occurrences of a subgraph $G$ whenever $G$ is guaranteed to have $O(n)$ occurrences. This would entail a new, constructive proof of Theorem 1.

Second, we would like to characterize the subgraphs occurring few times in minor-closed families other than those described in Theorem 2. In particular, the forbidden minors for graphs of bounded genus include graphs that are not 3-connected, so our characterization does not apply to these families. We would also like to know what happens when some $K_{b, c}$ is excluded for $b, c \geq 5$.

Third, perhaps we should investigate families of graphs that are not closed under minors. Any family of sparse graphs (more precisely, graphs with bounded arboricity) will contain only $O(n)$ cliques. But perhaps some other subgraphs occur few times. For instance, what are the subgraphs occurring few times in the $k$-page graphs [2]?

Finally, it might be interesting to characterize graphs occurring a number of times which is a non-linear function of $n$. For the families for which Theorem 2 applies, we can demonstrate a gap in the possible such functions: any subgraph $G$ occurs $\Omega(n)$ times, and the theorem tells us that $G$ either occurs $O(n)$ or $\Omega\left(n^{2}\right)$ times. For other families, there may be graphs that occur only $O(1)$ times (indeed, if $F$ excludes a minor consisting of $k$ disjoint copies of $G$, then $G$ occurs at most $k-1$ times in graphs of family $F$ ). If $G$ is a tree with $k$ leaves (other than a single edge), then it occurs $\Theta\left(n^{k}\right)$ times as a subgraph in other trees. Perhaps for planar graphs, a similar characterization can be described in terms of the tree of triconnected components. For sparse graphs (graphs with $O(n)$ edges) there can be at most $O\left(n^{3 / 2}\right)$ triangles [1], and more generally $O\left(n^{c / 2}\right)$ complete subgraphs $K_{c}$. Are there minor-closed families, and subgraphs in those families, such that the function describing the number of occurrences is similarly nonpolynomial?

\section{References}

[1] M. Bern and D. Eppstein. Mesh generation and optimal triangulation. Euclidean Geometry and Computers, D.A. Du and F.K. Hwang, eds., World Scientific, 1992.

[2] F. Bernhart and B. Kainen. The book thickness of a graph. J. Combinatorial Theory, Ser. B 27 (1979) 320-331.

[3] N. Chiba and T. Nishizeki. Arboricity and subgraph listing algorithms. SIAM J. on Computing 14 (1985) 210-223. 
[4] M. Chrobak and D. Eppstein. Planar orientations with low out-degree and compaction of adjacency matrices. Theoretical Computer Science 86 (1991) 243-266.

[5] M.B. Dillencourt and W.D. Smith. A linear-time algorithm for testing the inscribability of trivalent polyhedra. Proc. 8th ACM Symposium on Computational Geometry (1992) 177-185.

[6] C. Papadimitriou and M. Yannakakis. The clique problem for planar graphs. Information Processing Letters 13 (1981) 131-133.

[7] W.T. Tutte. A theory of 3-connected graphs. Proc. Koninklijke Nederlandse Akademie van Wetenschappen, Ser. A 64 (1961) 441-455. 